\title{
Vergleichbares Sicherheitsprofil wie die konservative Therapie
}

Fragestellung: Kann eine minimal invasive Implantation eines Spülkatheters gefolgt von einer Thrombolyse mit rt-PA zur Behandlung von intrazerebralen Blutungen eingesetzt werden?

Hintergrund: In westlichen Ländern machen intrazerebrale Blutungen $10-15 \%$ aller Schlaganfälle aus. Zwei große randomisierte Studien (STICH I und II) konnten keine Überlegenheit einer neurochirurgischen Entfernung der Blutung gegenüber einer optimalen konservativen Therapie belegen $[1,2]$. Daher ist es notwendig, andere Therapieverfahren zu entwickeln. Pathophysiologisch wird davon ausgegangen, dass intrazerebrale Blutungen zum einen über ihren Raumforderungseffekt wirken und zum anderen durch die beim Abbau der Blutung entstehenden Zerfallsprodukte eine Entzündungsreaktion hervorrufen. Die Arbeitsgruppe aus Baltimore von der Johns Hopkins University hat eine neue Behandlungsmethode entwickelt, die in einer ersten Sicherheitsstudie untersucht werden sollte.

Patienten und Methodik: MISTIE war eine offene Phase-II-Studie, die die Sicherheit und mögliche Wirksamkeit eines minimal invasiven neurochirurgischen Eingriffs mit der Platzierung eines Spülkatheters in die

Hanley DF, Thompson RE, Muschelli J et al. Safety and efficacy of minimally invasive surgery plus alteplase in intracerebral haemorrhage evacuation (MISTIE): a randomised, controlled, open-label, phase 2 trial. Lancet Neurol 2016; 15: 1228-37 Blutung und Gabe von rt-PA mit einer konservativen Therapie verglich. Die Studie wurde an 26 Krankenhäusern in den USA, Kanada, England und Deutschland durchgeführt. Eingeschlossen wurden Patienten mit einer nicht traumatischen in- trazerebralen Blutung mit einem Blutungsvolumen von $20 \mathrm{ml}$ oder mehr. Nach Anlage des Katheters wurde in der operativen Therapiegruppe rt-PA 0,3 mg oder 1,0 mg alle acht Stunden gegeben und das gelöste Blut dann über den Katheter abgesaugt. Die primären Endpunkte waren die 30-Tage-Sterblichkeit, die 7-Tage-Sterblichkeit, das Auftreten symptomatischer Blutungen nach 72 Stunden sowie intrakranielle Infektionen innerhalb von 30 Tagen. Sekundärer Endpunkt war der Outcome nach 180 und 365 Tagen, gemessen mit der modifizierten Rankin-Skala.

Ergebnisse: Zwischen Februar 2006 und April 2013 wurden 4.103 Patienten gescreent und letztendlich 96 randomisiert, davon 54 (56\%) in die operative und 42 (44\%) in die konservative Behandlungsgruppe. Nach 30 Tagen waren in der operativen Gruppe vier Patienten und in der konservativen Gruppe acht Patienten verstorben. Dieser Unterschied war statistisch nicht signifikant. Auch die Sterblichkeit innerhalb der ersten sieben Tage mit 0 versus 1 und die symptomatischen Blutungen mit 1 verus 5 waren nicht unterschiedlich. In der operativen Gruppe trat eine intrakranielle bakterielle Infektion auf. Asymptomatische Blutungen wurden in der operativen Gruppe mit 12 versus 3 signifikant häufiger beobachtet. Der funktionelle Outcome war nach 180 und 365 Tagen tendenziell besser für die Patienten, die operiert worden waren.

Schlussfolgerungen: Bezogen auf Sterblichkeit und symptomatische Blutungen hat ein minimal invasiver neurochirurgischer Eingriff mit Lyse der Blutung durch rt-PA ein gutes Sicherheitsprofil verglichen mit der konservativen Therapie. Beunruhigend ist allerdings die höhere Zahl asymptomatischer intrazerebraler Blutungen.

\section{- Kommentar von Hans-Christoph Diener, Essen}

\section{Ein insgesamt ernüchterndes Ergebnis}

Insgesamt ist das Ergebnis der MISTIE-Studie ernüchternd. Es handelte sich zwar primär um eine Sicherheitsstudie, man hät-

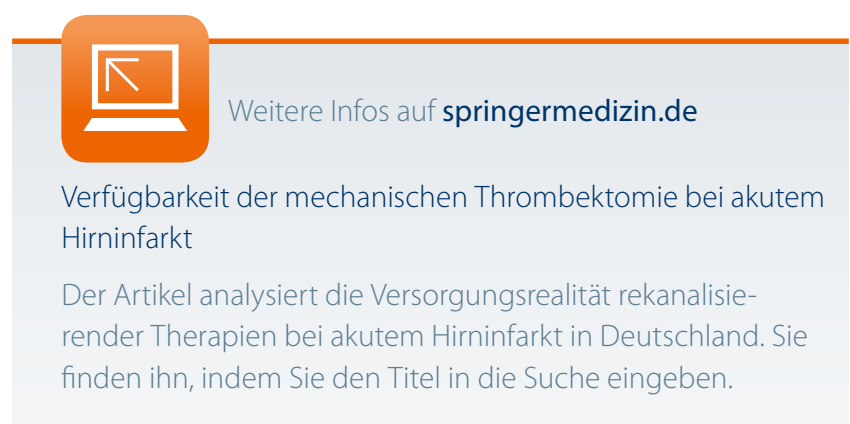

te allerdings einen Trend zugunsten des minimal invasiven neurochirurgischen Verfahrens erwartet. Man muss auch kritisch hinterfragen, ob eine Behandlungsmethode, bei der letztendlich 96 von 4.103 gescreenten Patienten tatsächlich behandelt werden, sich tatsächlich in die klinische Realität umsetzen lässt. Insgesamt war die Zahl der randomisierten Patienten zu gering, um eine mögliche Wirksamkeit zu belegen. Dies soll jetzt in der größeren MISTIE-III-Studie untersucht werden. Es bleibt allerdings zu hoffen, dass diese Studie nicht wieder sieben Jahre benötigt um ihre Rekrutierung zu beenden.

\footnotetext{
Referenzen:

1. Mendelow AD et al. Lancet 2005; 365: 387-97

2. Mendelow AD et al. Lancet 2013; 382: 397-408
} 